\title{
A case report on subarachnoid and intraventricular neurocysticercosis
}

\author{
Chen Shang ${ }^{1}$, Hong-Zhi Guan' ${ }^{1}$, Li-Ying Cui ${ }^{1}$, Bo Hou², Feng Feng ${ }^{2}$, Ding-Rong Zhong ${ }^{3}$ \\ ${ }^{1}$ Department of Neurology, Peking Union Medical College Hospital, China Academy of Medical Science, Beijing 100730, China. \\ ${ }^{2}$ Department of Radiology, Peking Union Medical College Hospital, China Academy of Medical Science, Beijing 100730, China. \\ ${ }^{3}$ Department of Pathology, Peking Union Medical College Hospital, China Academy of Medical Science, Beijing 100730, China.
}

\section{A B S T R A C T}

Neurocysticercosis is the most common central nervous system helminthic infection in humans. We hereby present a case combining two rare manifestations of neurocysticercosis: the subarachnoid and intraventricular forms. The patient presented with hydrocephalus and neurologic deficits and although endoscopic removal of the cysts and two cycles of postoperative cysticidal drugs resulted in resolution of symptoms, they later recurred. Ventriculoperitoneal shunt placement and a further cycle of albendazole plus dexamethasone led to substantial clinical improvement. Extraparenchymal neurocysticercosis may be challenging to diagnose and treat and is usually associated with a poorer prognosis. Clinicians should be aware of this condition.

Key words: Antiparasitic drug, extraparenchymal neurocysticercosis, intraventricular neurocysticercosis, neuroendoscopic surgery, subarachnoid neurocysticercosis

\section{INTRODUCTION}

Neurocysticercosis is caused by a human infestation of larvae of the tapeworm taenia solium and is considered the most common helminthic infection of the human central nervous system. The disease presents significant diagnostic and therapeutic challenges. It has heterogeneity in both clinical manifestation and therapeutic response $\mathrm{e}^{[1,2]}$ and mortality and morbidity rates remain high. ${ }^{[3]}$

Here, we describe a patient with subarachnoid and intraventricular neurocysticercosis who had hydrocephalus and neurologic deficits for several years, received successive surgical interventions, postoperative drug therapies, and finally, the placement of a ventriculoperitoneal (VP) shunt. Shunting proved, in this case, to be an effective treatment, and this may prompt its wider use in the treatment of the mixed form of extraparenchymal neurocysticercosis.

\begin{tabular}{|l|l|}
\hline \multicolumn{2}{|c|}{ Access this article online } \\
\hline Quick Response Code: & \\
\hline & Website: \\
\hline & www.nnjournal.net \\
\cline { 2 - 3 } & \\
\hline
\end{tabular}

\section{CASE REPORT}

A 56-year-old Chinese man was sent to our hospital for further evaluation and treatment. Approximately, 2 years prior to admission, he developed chronic headache with nausea intermittent dizziness, hearing loss, bradyphrenia, and mild lower limb weakness without remarkable findings on neurological examination besides bilateral optic disk edema cerebrospinal fluid (CSF) studies showed intracranial hypertension (opening pressure $\geq 330 \mathrm{mmH}_{2} \mathrm{O}$ ) and a CSF leukocytosis of $30 \times 10^{6} / \mathrm{L}$ ( $90 \%$ lymphocytes, $5 \%$ monocytes, $3 \%$ neutrophils, and $2 \%$ eosnophils). Brain magnetic resonance imaging (MRI) showed widened sulci and cisterns bilaterally, as well as enlargement of the subarachnoid spaces. He stated that he used to eat under-cooked meat. Serum immunoblot for the anticysticercal antibodies was equivocal. The assay of CSF anticysticercal antibodies was positive, and a presumptive diagnosis of neurocysticercosis causing significant hydrocephalus was made.

A transfrontal transventricular endoscopic excision and third ventriculostomy were undertaken to relieve the clinical symptoms. Thickening of the arachnoid membrane underneath the third ventricle was found and upon fenestration of the arachnoid to the basic cistern, a multitude of whitish cystic structures with

Corresponding Author: Dr. Hong-Zhi Guan, Department of Neurology, Peking Union Medical College Hospital, China Academy of Medical Science, No. 1 Shuaifuyuan Wangfujing, Dongcheng District, Beijing 100730, China. E-mail: guanzhaoduoduo@hotmail.com 
thin-walled membrane were seen. These yellow-spotted, irregularly-shaped cysts were intimately attached to the arachnoid or the basilar artery. Their direct visualization led to the confirmation of the diagnosis of subarachnoid neurocysticercosis. Subsequently, cysts were gently grasped and removed for histopathological evaluation, which revealed evidence of degenerative changes and an inflammatory reaction within the walls, mediated by nuclear macrophage and eosinophil infiltration [Figure 1]. The patient then received two cycles of antihelmintic therapies with albendazole and corticosteroid. This resulted in complete resolution of the patient's symptoms and he returned to his normal daily activities.

Approximately 4 months after the surgery, the patient had a recurrence of the same symptoms. Brain MRI again revealed evidence of hydrocephalus. A VP shunt was placed, resulting in no obvious improvement of clinical symptoms.

One year later, the patient reported new symptoms of motor deficiency and urinary incontinence, which led to his admission to our hospital. Physical examination showed he was drowsy, but oriented. He demonstrated full strength in his arms but decreased strength in his legs. The finger-nose and heel-knee-tibia tests lacked accuracy on both sides and Romberg's sign was positive. All the left-sided deep tendon reflexes were pathologically brisk. Babinski's sign was negative on both sides. The patient had no sensory deficits and no obvious meningismus.

Computed tomography (CT) revealed persistent ventricular dilation. A lumbar puncture was performed on this patient, and the opening pressure was now normal $\left(160 \mathrm{mmH}_{2} \mathrm{O}\right)$. CSF studies also demonstrated a significantly increased number of white blood cells with a predominance of lymphocytes, an elevated protein level $(0.81 \mathrm{~g} / \mathrm{L})$, and a decreased glucose concentration $(0.1 \mathrm{mmol} / \mathrm{L})$. Further evaluations for tuberculosis, bacteremia, fungal infection, and autoimmune processes were negative. Brain MRI indicated multiple small cysts containing a CSF-like

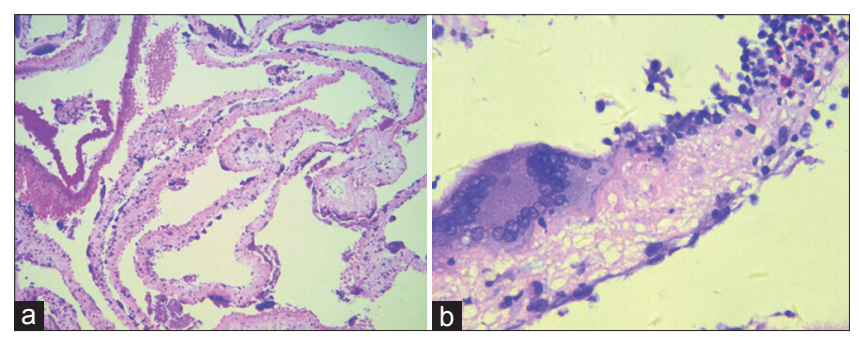

Figure 1: Hematoxylin and eosin stained low power field image (a) cysticercus larva. The multiple cysts of cysticercus larva underwent degenerative changes. High power field image (x100); (b) the wall of cysts infiltrated by multiple nuclear macrophages and eosinophils (x200) signal within both lateral ventricles that did not enhance with contrast [Figure 2]. A third cycle of albendazole and corticosteroid was administrated, which produced an improvement in cognitive status, and lower limb power and coordination.

\section{DISCUSSION}

Neurocysticercosis, caused by larvae of the tapeworm taenia solium, is the most common form of parasitic brain disease globally. ${ }^{[1,4]}$ It can occur in intraparenchymal, intraventricular, subarachnoid, or mixed forms. ${ }^{[5]}$

A major characteristic of neurocysticercosis is heterogeneity, with the clinical manifestations dependent on the localization, number, and evolutional stage of the parasites, as well as the intensity of the inflammatory reaction. Patients with neurocysticercosis may be asymptomatic, or present with a wide variety of symptoms. ${ }^{[1]}$ Typical CSF findings of neurocysticercosis include moderate mononuclear pleocytosis, mainly of lymphocytes and elevated protein concentrations, ranging from $0.5 \mathrm{~g} / \mathrm{L}$ to $2.0 \mathrm{~g} / \mathrm{L}$. In most cases, CSF glucose concentrations are normal or moderately decreased. ${ }^{[4]}$ These CSF abnormalities are not present in all cases, and so cannot be used as definite diagnostic criteria. ${ }^{[3]}$ Usually, neuroimaging findings of extraparenchymal cysticerci are subtle: the cystic walls are thin, there is often an absence of pathognomonic scolices, central cysts are isointense to CSF and they do not enhancement after contrast administration. ${ }^{[3]}$ Detection of specific serum or CSF antibodies plays a helpful role in the diagnosis of cerebral cysticercosis, but it cannot differentiate between viable and degenerated parasites and is unable to confirm CNS localization. ${ }^{[6]}$ The diagnosis in this case was made with the help of direct endoscopic visualization and histologic demonstration. Since there exist enough

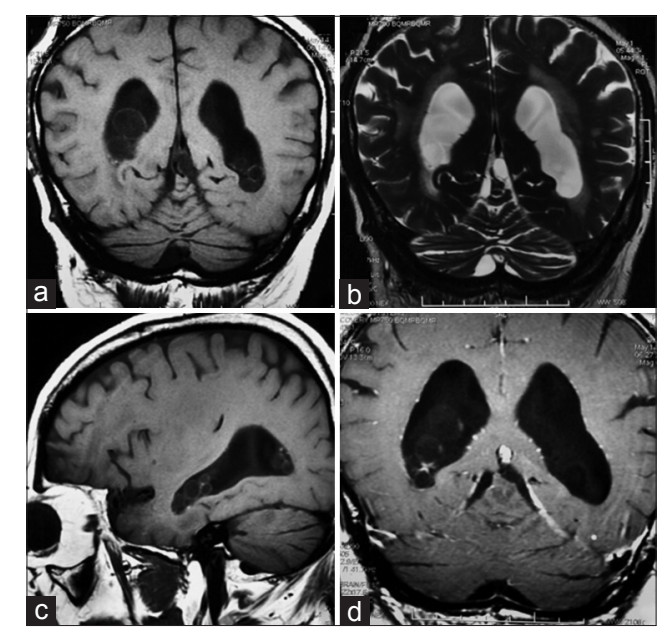

Figure 2: Brain magnetic resonance imaging showed multiple small cysts with cerebrospinal fluid-like signal inside within the lateral ventricles (a and c) T1-weighted, (b) T2-weighted and no enhancement (d) T2-weighted with contrast-enhanced) 
space, cysts in nonconfining extraparenchymal areas can grow into interconnected grape-like clusters, known as racemose neurocysticercosis. ${ }^{[5]}$ The parasite in the extraparenchymal space degenerates owing to the continuous CSF ingress into the vesicles, ${ }^{[3]}$ forming a hyaline mass. Histopathologically, this is a granulomatous lesion infiltrated by many multinucleate giant macrophages. ${ }^{[4]}$

Treatment should be individualized, ${ }^{[4]}$ particularly for patients with mixed forms of neurocysticercosis. Since extraparenchymal neurocysticercosis is associated with a poorer prognosis, there was a consensus toward more aggressive management. ${ }^{[7]}$ The surgical option was attractive because drug penetration into the ventricular and subarachnoid spaces is much lower when compared with that into the brain parenchyma. Our patient received endoscopic therapy, which resulted in rapid and safe reduction of the parasite burden. However, given that not all the cysticercus can be removed completely during the surgery, antihelminthic drugs are still required. ${ }^{[8]}$ Albendazole is the preferred choice because it has a superior penetration of the subarachnoid space, reaching higher concentration in the CSF than alternative agents and shows efficacy in treating both subarachnoid and ventricular cysts. ${ }^{[9]}$ Between the second and 5 th days of antiparasitic therapy, there is usually an exacerbation of neurological symptoms attributed to local inflammation due to the larval death. ${ }^{[7]}$ For this reason, corticosteroids are given with parasiticidal drugs. Management of elevated intracranial pressure secondary to neurocysticercosi is also a priority. In order to make an improvement to the CSF circulation, this patient underwent endoscopic third ventriculostomy (ETV) and VP shunt replacement. It has been reported ETV could decrease the shunt failure rate from $36 \%$ to $8 \%{ }^{[10]}$

The optimal duration of anti-parasitic treatment for extraparenchymal neurocysticercosis is not known. ${ }^{[7]}$
Further work is required in patients with subarachnoid and ventricular neurocysticercosis to establish the roles of higher doses of albendazole, combined antiparasitic drugs, prolonged drug courses, repeated cycles, surgical interventions, and multidisciplinary collaborative working. ${ }^{[2,3]}$

\section{REFERENCES}

1. Carpio A, Fleury A, Hauser WA. Neurocysticercosis: five new things. Neurol Clin Pract 2013;3:118-25.

2. Del Brutto OH. Neurocysticercosis. Continuum (Minneap Minn) 2012;18:1392-416.

3. Fleury A, Carrillo-Mezo R, Flisser A, Sciutto E, Corona T. Subarachnoid basal neurocysticercosis: a focus on the most severe form of the disease. Expert Rev Anti Infect Ther 2011;9:123-33.

4. Carpio A. Neurocysticercosis: an update. Lancet Infect Dis 2002:2:751-62

5. Das RR, Tekulve KJ, Agarwal A, Tormoehlen LM. Racemose neurocysticercosis. Semin Neurol 2012;32:550-5.

6. Fleury A, Escobar A, Fragoso G, Sciutto E, Larralde C. Clinical heterogeneity of human neurocysticercosis results from complex interactions among parasite, host and environmental factors. Trans $R$ Soc Trop Med Hyg 2010;104:243-50.

7. Garcia HH, Evans CA, Nash TE, Takayanagui OM, White AC Jr, Botero D, Rajshekhar V, Tsang VC, Schantz PM, Allan JC, Flisser A, Correa D, Sarti E, Friedland JS, Martinez SM, Gonzalez AE, Gilman RH, Del Brutto OH. Current consensus guidelines for treatment of neurocysticercosis. Clin Microbiol Rev 2002;15:747-56.

8. Wu W, Jia F, Wang W, Huang Y, Huang Y. Antiparasitic treatment of cerebral cysticercosis: lessons and experiences from China. Parasitol Res 2013;112:2879-90.

9. Carpio A. Albendazole therapy for subarachnoid cysticerci: clinical and neuroimaging analysis of 17 patients. $J$ Neurol Neurosurg Psychiatry 1999;66:411-2.

10. Torres-Corzo JG, Tapia-Perez JH, Vecchia RR, Chalita-Williams JC, Sanchez-Aguilar M, Sanchez-Rodriguez JJ. Endoscopic management of hydrocephalus due to neurocysticercosis. Clin Neurol Neurosurg 2010;112:11-6.

Cite this article as: Shang C, Guan HZ, Cui LY, Hou B, Feng F, Zhong DR. A case report on subarachnoid and intraventricular neurocysticercosis. Neuroimmunol Neuroinflammation 2015;2(3):171-3.

Source of Support: Nil. Conflict of Interest: No.

Received: 15-12-2014; Accepted: 10-06-2015 